\title{
Puerarin and betahistine treatment of vertebrobasilar ischemia vertigo: A meta-analysis of randomized controlled trials
}

\author{
YAN-YAN CHEN $^{1}$, WEN CHEN ${ }^{2}$, QING ZHANG ${ }^{1}$, HUI LI $^{1}$, \\ YE-WEN ZHANG ${ }^{1}$, QIAN KANG ${ }^{1}$, YI LAN $^{1}$ and QING WU ${ }^{1}$ \\ ${ }^{1}$ School of Chinese Materia Medica, Beijing University of Chinese Medicine, Beijing 100102; \\ ${ }^{2}$ Key Laboratory of Food Nutrition and Safety, Tianjin University of Science and Technology, \\ Ministry of Education, Tianjin 300457, P.R. China
}

Received November 4, 2014; Accepted November 5, 2015

DOI: $10.3892 / \mathrm{etm} .2016 .3004$

\begin{abstract}
The present meta-analysis aimed to evaluate the effectiveness and safety of puerarin co-treatment with betahistine in treating vertebrobasilar ischemia (VBI) vertigo. A total of 6 medical databases were searched, identifying randomized controlled trials (RCTs) of VBI vertigo performed until August 2014 that investigated a combined treatment of puerarin with betahistine or with other conventional drugs. The quality of the literature was evaluated using the Cochrane Collaboration's tool for assessing risk of bias, and Rev Man 5.0 software was used for statistical analysis and evaluation. The present study included 7 RCTs, involving a total of 664 subjects, and revealed a statistically significant increase in efficacy between the control and the experimental group (odds ratio [OR], 4.99; 95\% confidence interval [CI], 3.05 to 8.15). The average blood flow velocity within the vertebrobasilar arteries increased following treatment with puerarin and betahistine compared with that of the control groups (OR, 7.59; 95\% CI, 6.19 to 9.00); however, no difference was detected between these groups in the average flow velocity within the left vertebral artery (OR, 6.17; 95\% CI, 5.22 to 7.13). The frequency of adverse reactions in the experimental group was lower (OR, $0.75 ; 95 \%$ CI, 0.32 to 1.77 ) compared with the control group. Combined puerarin and betahistine regimens were more effective in treating VBI vertigo compared with other, conventional drugs; effectively alleviating the associated symptoms, including dizziness and increased average blood flow velocity within the vertebrobasilar arteries, without causing an increased number of serious side effects. However, the efficacy and safety of puerarin and betahistine use in treating VBI vertigo requires additional investigation.
\end{abstract}

Correspondence to: Professor Qing Wu, School of Chinese Materia Medica, Beijing University of Chinese Medicine, 11 North Third Ring Road East, Beijing 100102, P.R. China

E-mail: qwu@vip.sina.com

Key words: puerarin, betahistine, vertebrobasilar ischemia vertigo, randomized controlled trials, meta-analysis

\section{Introduction}

Vertebrobasilar ischemia (VBI) vertigo is an otolaryngological disease that is common in elderly patients. The cerebellum and brain stem rely on blood flow from the vertebrobasilar arteries; however, when lesions develop in the vertebrobasilar arteries, poor blood supply to the brain leads to dizziness, referred to as central vestibular vertigo (1). Lesions within the vestibular nuclei, brain stem, cerebellum and temporal lobe are the predominant cause of central vestibular vertigo, which presents with a continuous and progressive set of symptoms (2). VBI vertigo accounts for $>20 \%$ of defective cerebrovascular disease (3), and is a common cause of vertigo in senile patients, $\sim 5 \%$ of which develop complete cerebral apoplexy (4). Clinical manifestations, including dizziness, nausea and vomiting, often occur, and resistance to treatment is characteristic of VBI vertigo (5). The primary symptom in $50-80 \%$ of patients with VBI is dizziness (6) with tinnitus and hearing loss. VBI has multiple risk factors, including increased platelet aggregation rate, high blood pressure, high blood viscosity, hyperlipidemia, cervical spondylosis, bradycardia and low blood sugar (1). During an acute episode, patients suffer from dizziness combined with nausea, vomiting, instability and difficulty of movement, which should be treated rapidly. Generally comprehensive measures, which aim to reduce artery spasm, improve brain microcirculation, improve brain tissue oxygen supply and restore the brain function, as well as intramuscular or intravenous injection or reducing oral drugs are considered to be the primary measures for the treatment of VBI vertigo.

Kudzu root, which is known as 'Ge gen' in traditional Chinese medicine, is the dried root of Pueraria lobata (Willd.) Ohwi; a perennial, leguminous vine that is native to southeast Asia. For >2,000 years, kudzu root has been used as a herbal medicine in the treatment of fever, acute dysentery, diarrhoea, diabetes and cardiovascular disease, containing isoflavonoids and triterpenoids as its predominant active constituents (7). Puerarin is a flavonoid glycoside, and is the major bioactive ingredient and the most abundant secondary metabolite present in the root of P. lobata (Willd.) Ohwi. Puerarin is available in numerous foods, is used in alternative medicine (8) and is able to expand the cerebrovascular and coronary arteries (9). This has been hypothesized to 
produce numerous downstream effects, including reducing myocardial oxygen consumption, inhibiting platelet aggregation, improving erythrocyte deformability, eliminating oxygen free radicals and improving the function of blood rheology, thereby ameliorating the insufficient vertebrobasilar blood supply (10). Puerarin is increasingly employed in treatment of cardiovascular and cerebrovascular diseases, diabetes with its associated complications (11), Parkinson's and Alzheimer's disease (12), osteonecrosis (13), cancer and endometriosis (14). The effectiveness of puerarin in these diverse medicinal contexts may be due to its numerous suggested pharmacological functions, which potentially include vasodilative (15), cardioprotective (16), neuroprotective (17), antioxidative (18), anticancer (19) and anti-inflammatory effects, in addition to pain alleviation, increased osteogenesis (20), reduced alcohol intake and decreased resistance to insulin (8).

Previous studies have increasingly reported that combining puerarin with betahistine or with other, conventional drugs may enhance its effects in relieving dizziness and other side effects in patients with VBI (21-29). Furthermore, the treatment of insufficient blood supply within the vertebrobasilar arteries using a single drug has its limitations, since different treatments should be adopted according to the cause of insufficient blood supply, which can be complex. For instance, platelet aggregation inhibitors, vascular expansion agents, cerebral vasodilator and albumin light quantum therapy can be used for atherosclerosis, while cervical traction can be used for cervical hyperostosis. Systematic consideration of all appropriate evidence on use of a particular factor, such as by meta-analysis, is therefore required (30). This may be used to identify relevant studies, extract relevant data, appraise study methods and statistically evaluate the associated studies $(31,32)$. To the best of our knowledge, the majority of the studies regarding puerarin and betahistine combined treatment of VBI vertigo has not been rigorously investigated. Therefore, an evaluation of the efficacy and safety of this combination of traditional Chinese medicine and modern treatment for VBI vertigo is required. In the present study, a systematic evaluation of randomized controlled trials (RCTs) of puerarin and betahistine combinatorial treatment of VBI vertigo was conducted in order to assess its overall effectiveness and safety and to provide the basis for subsequent clinical studies regarding its application.

\section{Materials and methods}

Search strategy. Electronic databases were searched, including entries from the following periods: Chinese National Knowledge Infrastructure (CNKI; www.cnki.net/), January 1987 to August 2014; Wanfang Med Online Database (WMOD; www.wanfangdata.com.cn/), April 1998 to August 2014; Chinese VIP Information (VIP; www.cqvip.com/), February 1989 to August 2014; PubMed (www.pubmed.cn/), November 1967 to August 2014; the Cochrane Central Register of Controlled Trials (www.cochranelibrary.com/), March 1999 to August 2014; and the Chinese Biomedical Literature Database (CBM; www.sinomed.ac.cn/), October 1978 to August 2014. The search terms used were 'puerarin', 'betahistine', 'betahistine hydrochloride)' and 'vertebrobasilar ischemia vertigo'. No language restriction was applied.
Study selection and eligibility criteria. The titles and abstracts of potentially relevant references were identified through the literature search, checked to determine their suitability with regard to the meta-analysis inclusion criteria and independently reviewed by two reviewers. Discrepancies regarding whether to include a study were resolved by consensus with an additional investigator. The present study only considered RCTs using puerarin and betahistine treatment of VBI vertigo, irrespective of language, whether the RCT was from a periodical or magazine, publishing year and database.

Inclusion criteria were as follows: i) Studies were RCTs, regardless of whether these were blinded studies; ii) sample sizes $\geq 20$ participants; iii) based on strict diagnostic criteria of VBI vertigo, according to the guidelines of the World Health Organization (33), but regardless of age and gender; iv) patients in the intervention group were co-administered an injection of puerarin (such as Chia Tai-Tianqing Pharmaceutical Group Co., Ltd., Jiangsu, China) and betahistine (such as Yabao Pharmaceutical Group Co., Ltd., Beijing, China), administered via multiple routes. The control groups were administered a routine treatment, such as betahistine, compound Danshen injection (which consists of extracts of Salvia miltiorrhiza and Dalbergiae odoriferae), sibelium or low molecular weight dextran, regardless of manufacturer, preparation form, dose or duration of treatment.

Selected outcomes. The primary outcome measure was clinical efficacy, which was expressed as follows: Cured, when signs and symptoms disappear entirely, and transcranial Doppler (TCD) indicates that the vertebral/basilar artery blood supply returns to normal; excellent, when symptoms, such as dizziness, are significantly reduced and patients can have a regular life, while TCD displays that the vertebral/basilar artery blood supply is improved by $>60 \%$; valid or effective, in cases when the symptoms are slightly reduced slightly, and TCD displays improvement of $>40 \%$ in the vertebral/basilar artery blood supply; and invalid, in cases when the symptoms are not evidently relieved, and TCD displays improvement of $<40 \%$ in the vertebral/basilar artery blood supply. The secondary outcome measure was the increase in average blood flow velocity of the vertebrobasilar arteries (the basilar artery and the left and right vertebral arteries). The final outcome considered was the incidence of adverse reactions (including diarrhea, nausea and polypnea) of the patients in the two groups.

Data extraction and management. Data from each review were extracted by two independent authors in accordance with the aforementioned criteria, using a self-developed data extraction form in Microsoft Excel 2010 (Microsoft Corporation, Redmond, WA, USA), and the data were validated by a third author. Disagreements were resolved by discussion between the two reviewers, and by the opinion of a third reviewer if necessary. Details noted from the studies included study design, treatment duration, the age of participants, the number of participants and assessments of the aforementioned outcomes.

Trial quality assessment. In order to reduce subjective bias, the two authors independently appraised the methodological quality of each review in accordance with the Cochrane handbook (34) and quality assessment of the RCTs was conducted using 


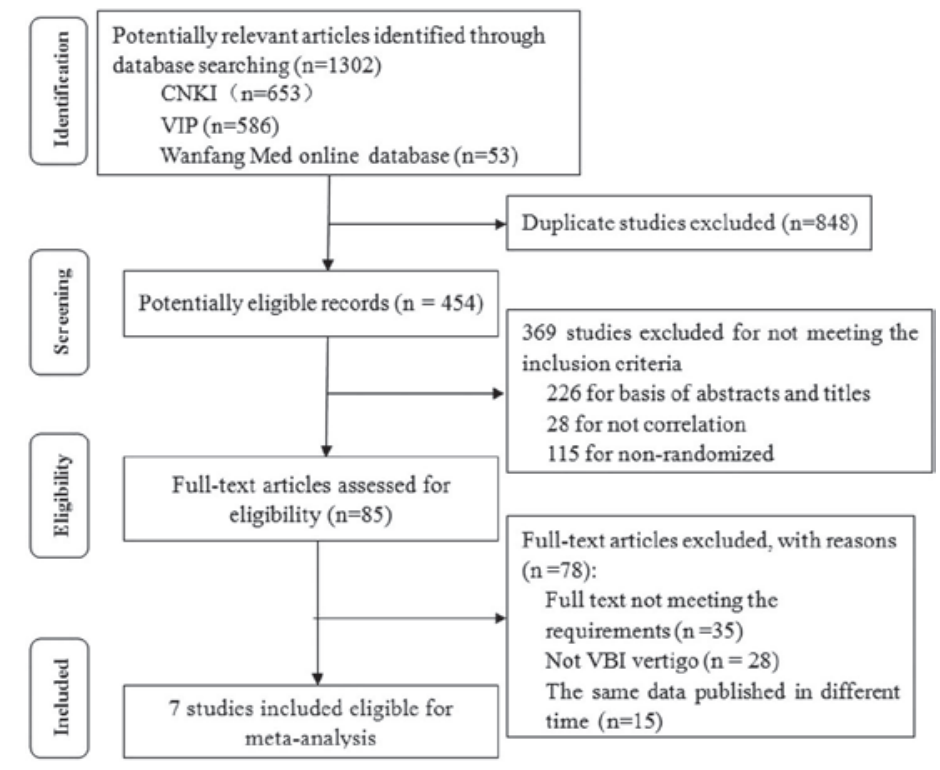

Figure 1. Study selection process for the meta-analysis.

Jadad's validated score (35). Quality assessment included evaluation of randomization, blinding of assessors to the treatment groups, allocation concealment regardless of the results of the allocation and intentional analysis. Each of these criteria was worth one point; a study scoring $>3$ points was considered to be high-quality, while $<3$ points indicated a low-quality study.

Statistical analysis. Statistical analyses were conducted using RevMan version 5.0.18 (The Nordic Cochrane Centre, The Cochrane Collaboration, Copenhagen, Denmark) software. An examination of odds ratio (OR) was used to analyze efficacy of the numerical/quantitative data, the interval size is expressed using $95 \%$ confidence interval (CI) and the heterogeneity of the results was assessed using a $\chi^{2}$ test.

The studies were initially tested for clinical heterogeneity. A fixed-effects model was used upon detection of statistical homogeneity (indicated by a P-value of $>0.1$ or an $\mathrm{I}^{2}$ value $<50 \%$ ); however, a random effect model would be used upon detection of statistical heterogeneity (indicated by a P-value of $<0.1$ or an $\mathrm{I}^{2}$ value $>50 \%$ ) (36) or when no clinical heterogeneity or no statistically significant difference existed in the trials. If there was clinical and methodological heterogeneity, meta-regression, subgroup analysis and sensitivity analysis were conducted (37). In the presence of clear heterogeneity, only a descriptive analysis of the data was made. Binary classification variables were expressed using OR, continuous variables were expressed using the weighted mean differences or standardized mean differences and effect size was expressed by the $95 \%$ CI. $\mathrm{P}<0.05$ was considered to indicate a statistically significant difference. Publication bias was assessed by a funnel plot (38).

\section{Results}

\section{Study selection}

Description of studies. Initial searches yielded 1,302 studies (653 from CNKI, 586 from VIP, 53 from Wanfang Med Online Database) in accordance with the aforementioned search strategy. Following removal of 848 duplicate studies, the abstracts of 454 studies were evaluated. Subsequent to the evaluation of the abstract and title of each study, 369 studies were excluded as they did not meet the inclusion criteria. The full text of each of the remaining 85 trials was read, and 35 trials were excluded in accordance with the study criteria; 28 of these trials did not examine VBI vertigo and 15 trials included repetition of data, leaving 7 trials remaining. The results of the present meta-analysis are therefore based on information from 7 RCTs (21-27), which included a total of 664 patients. These RCTs were all conducted in China and published in Chinese. Fig. 1 presents a flow chart of the trial selection process.

Characteristics of trials included. The characteristics of the included 7 RCTs are summarized in Table I. Data regarding patient age, gender, duration of treatment, the number of participants and adverse reactions are provided for all trials. The average age of the participants ranged from 53.0-64.32 years, the duration of treatment ranged from 7-14 days and the number of patients in the studies ranged from 23-69, with a total of 664 patients (333 patients in treatment groups) across the 7 RCTs. The available data revealed that studies predominantly included male patients. Drugs for the intervention group included puerarin injection in addition to betahistine hydrochloride tablets $(21,24)$, betahistine mesylate $(22,23,26,27)$, which was administered orally or through intravenous drip, or betahistine injection (25). Conventional drugs administered to the control groups included Compound Danshen injection with low molecular weight dextran $(21,22,25,26)$, Compound Danshen injection only (23), sibelium capsule with low molecular weight dextran (24) or betahistine mesylate only (27). Publication year of the 7 RCTs ranged between 2007-2013, and trials published in 2010-2013 represented 4/7 of these trials (57.14\%). With regard to the evaluated outcomes, the reviews assessed clinical symptoms and signs $(4 / 7,57.14 \%)$, vertigo symptoms and signs $(3 / 7,42.86 \%)$ and alterations in hemorheology $(2 / 7,28.57 \%)$. Adverse reactions were not reported in 3 trials $(21,23,27)$.

Methodological quality of included trials. In the 7 studies included, 1 trial (26) mentioned that the intervention and control groups were generated using a random digits table; 
Table I. Characteristics of the included studies.

\begin{tabular}{|c|c|c|c|c|c|c|c|c|}
\hline $\begin{array}{l}\text { Author, } \\
\text { year }\end{array}$ & $\begin{array}{l}\text { Group: no. patients } \\
\text { (male/female) }\end{array}$ & $\begin{array}{c}\text { Average } \\
\text { age (years) }\end{array}$ & $\begin{array}{l}\text { Intervention } \\
\text { group }\end{array}$ & $\begin{array}{l}\text { Control } \\
\text { group }\end{array}$ & $\begin{array}{l}\text { Duration } \\
\text { (days) }\end{array}$ & $\begin{array}{l}\text { Outcome } \\
\text { measures }\end{array}$ & $\begin{array}{l}\text { Adverse } \\
\text { reactions }\end{array}$ & Ref. \\
\hline $\begin{array}{l}\text { Sun and Dang, } \\
2007\end{array}$ & $\begin{array}{c}69(22 / 47) \\
\text { C: } 69(28 / 41)\end{array}$ & $\begin{array}{l}59 \\
59\end{array}$ & $\mathrm{PI}+\mathrm{BHT}$ & CDI + LMD & 7 & A & Not mentioned & $(21)$ \\
\hline $\begin{array}{l}\text { Yue et al., } \\
2013\end{array}$ & $\begin{array}{l}\text { T: } 46(26 / 20) \\
\text { C: } 45(26 / 19)\end{array}$ & $\begin{array}{l}56 \pm 7 \\
56 \pm 7\end{array}$ & $\mathrm{PI}+\mathrm{BM}$ & CDI + LMD & 7 & A & Dizziness, nausea & $(22)$ \\
\hline $\begin{array}{l}\text { Yang, } \\
2010\end{array}$ & $\begin{array}{l}\text { T: } 23(13 / 10) \\
\text { C: } 23(14 / 9)\end{array}$ & $\begin{array}{l}57 \pm 8 \\
57 \pm 9\end{array}$ & $\mathrm{PI}+\mathrm{BM}$ & CDI & 14 & $\mathrm{~A}, \mathrm{~B}$ & Not mentioned & (23) \\
\hline $\begin{array}{l}\text { Wang, } \\
2011\end{array}$ & $\begin{array}{l}\text { T: } 30(21 / 9) \\
\text { C: } 30(20 / 10)\end{array}$ & $\begin{array}{l}55 \\
53\end{array}$ & $\mathrm{PI}+\mathrm{BHT}$ & $\mathrm{SC}+\mathrm{LMD}$ & 14 & A,B & Skin itching & (24) \\
\hline $\begin{array}{l}\text { Xie, } \\
2008\end{array}$ & $\begin{array}{l}\text { T: } 79(45 / 34) \\
\text { C: } 79(47 / 32)\end{array}$ & $\begin{array}{c}64 \pm 10 \\
64 \pm 8\end{array}$ & $\mathrm{PI}+\mathrm{BI}$ & CDI + LMD & 14 & A,B & $\begin{array}{l}\text { Facial flush, heart } \\
\text { palpitations, dry } \\
\text { mouth, abdominal } \\
\text { discomfort, nausea, } \\
\text { fever }\end{array}$ & (25) \\
\hline $\begin{array}{l}\text { Guo, } \\
2012\end{array}$ & $\begin{array}{l}\text { T: } 43(24 / 19) \\
\text { C: } 44(25 / 19)\end{array}$ & $\begin{array}{l}56 \pm 7 \\
56 \pm 7\end{array}$ & $\mathrm{PI}+\mathrm{BM}$ & CDI + LMD & 7 & $\mathrm{~A}, \mathrm{~B}$ & Diarrhea, nausea & (26) \\
\hline $\begin{array}{l}\text { Lei, } \\
2008\end{array}$ & $\begin{array}{l}\text { T: } 43(14 / 29) \\
\text { C: } 40(12 / 28)\end{array}$ & $\begin{array}{l}57 \\
58\end{array}$ & $\mathrm{PI}+\mathrm{BM}$ & $\mathrm{BM}$ & 7 & A & Not mentioned & (27) \\
\hline
\end{tabular}

Average age values were rounded to the nearest whole number. T, intervention group; C, control group; PI + BHT, puerarin injection plus betahistine hydrochloride tablets; PI + BM, puerarin injection plus betahistine mesylate. PI + BI, puerarin injection plus betahistine injection. CDI + LMD, compound danshen injection plus low molecular weight dextran. CDI, compound danshen injection. SC + LMD, sibelium capsule plus low molecular weight dextran; A, progress of clinical symptoms and signs; B, changes to hemorheology index, according to Jadad's validated score (35).

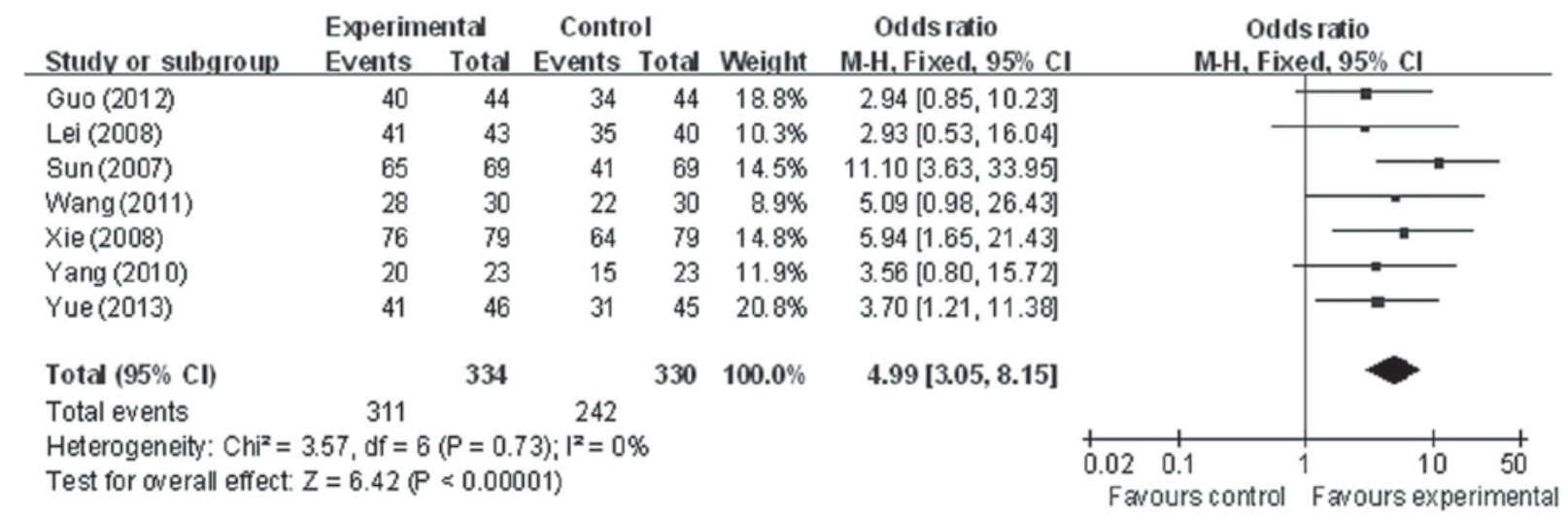

Figure 2. Therapeutic efficacy of puerarin combined with betahistine in treating vertebrobasilar ischemia vertigo. CI, confidence interval; M-H, Mantel-Haenszel method.

however, the remaining articles did not mention a randomization method. Allocation concealment and intentional analysis were not mentioned in any of the studies included in the present meta-analysis.

\section{Primary outcomes}

Evaluation of therapeutic efficacy. Clinical efficacy (determined as cured, excellent, effective or invalid) was the primary factor studied, and the total effective rate was reported in all 7 studies (21-27). Analysis revealed statistical homogeneity of the total effective rate within the present studies $\left(\chi^{2}=3.57\right.$; $\mathrm{P}=0.73 ; \mathrm{I}^{2}=0 \%$ ), leading to adoption of the fixed-effect model for meta-analysis. However, a statistically significant difference was identified in total efficacy rate between the intervention and control groups (OR, 4.99; 95\% CI, 3.05 to 8.15) (Fig. 2).
Assessment of the average blood flow velocity of the vertebrobasilar arteries. Inevitably, studies brought together in a systematic review will differ; any kind of variability among studies in a systematic review may be termed heterogeneity (39). Increase in the average blood flow velocity of the vertebrobasilar arteries (the basilar artery and left and right vertebral arteries) of patients with VBI vertigo was reported in 2 studies $(23,26)$. A $\chi^{2}$ test was performed to determine whether there were significant differences in homogeneity amongst the studies; this indicated no significant homogeneity in the change observed in blood flow velocity of the vertebrobasilar arteries $\left(\chi^{2}=2.11 ; \mathrm{P}=0.15 ; \mathrm{I}^{2}=53 \%\right)$. A meta-analysis was therefore performed using a fixed-effects model. Statistical analyses revealed a significant increase in blood flow velocity of vertebrobasilar arteries between the control 


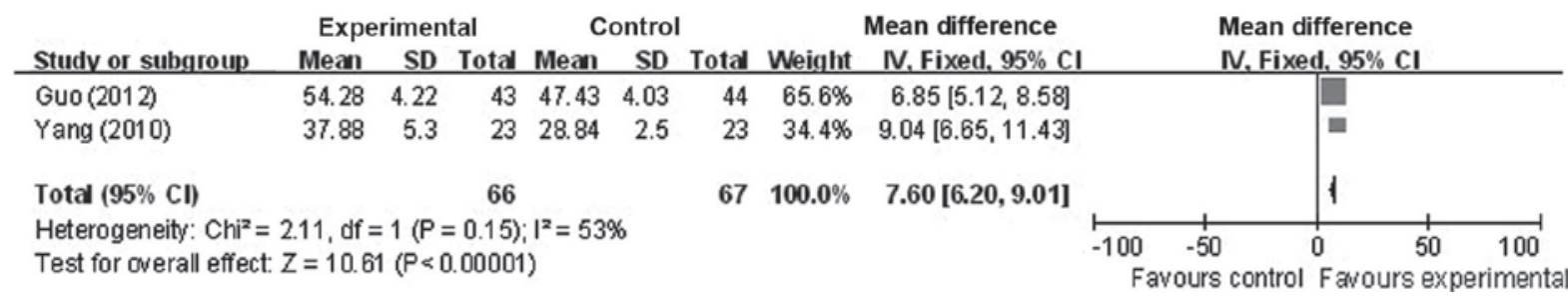

Figure 3. Effect of puerarin combined with betahistine on blood velocity in the basilar artery. SD, standard deviation; CI, confidence interval.

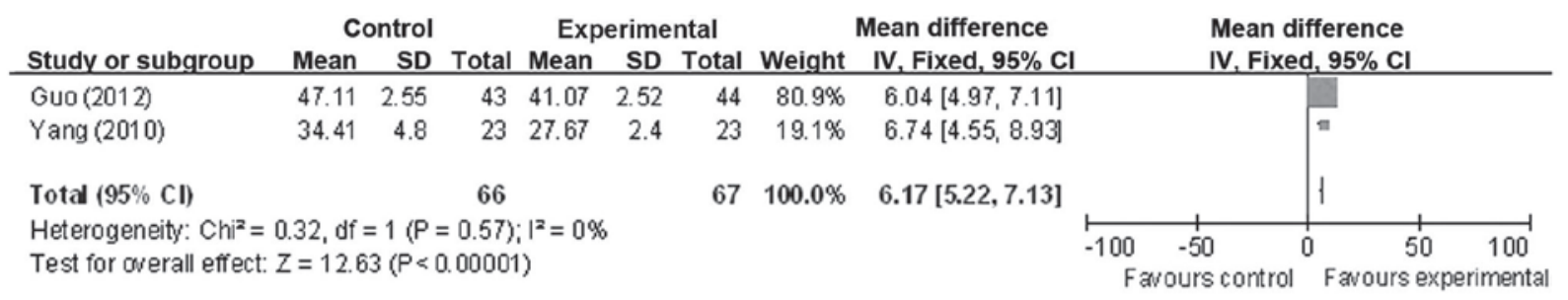

Figure 4. Improvement in the blood flow velocity of the left vertebral artery. SD, standard deviation; CI, confidence interval.

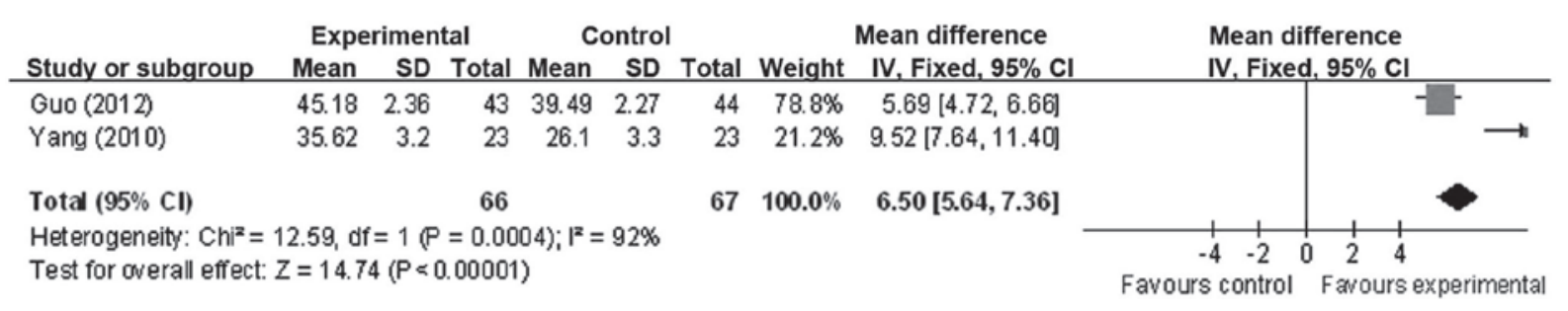

Figure 5. Improvement in the blood flow velocity of the right vertebral artery. SD, standard deviation; CI, confidence interval.

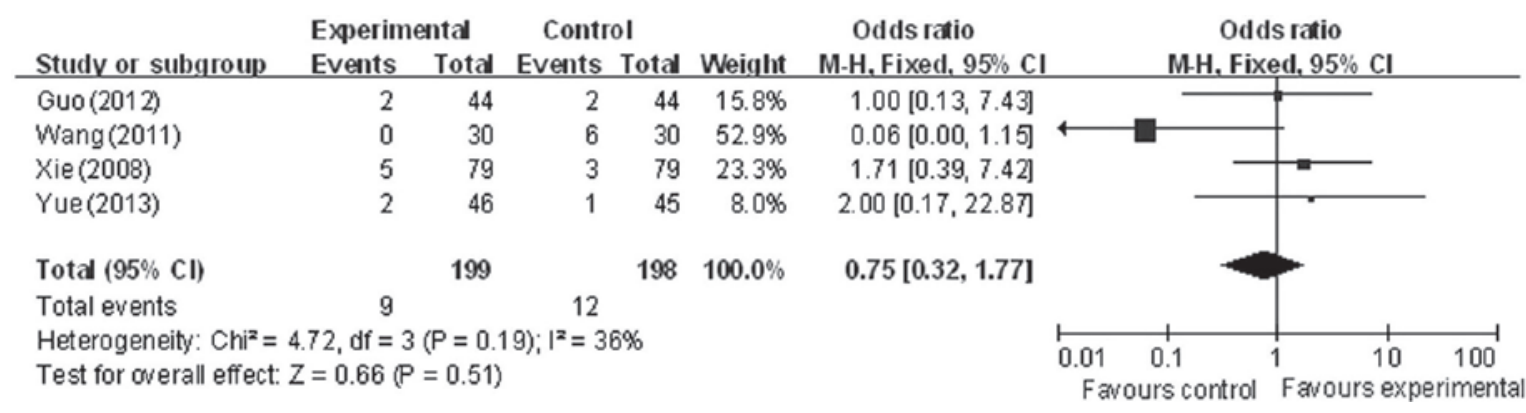

Figure 6. Incidence of adverse reactions to puerarin combined with betahistine in the treatment of vertebrobasilar ischemia vertigo. CI, confidence interval; M-H, Mantel-Haenszel method.

and intervention groups (OR, 7.60; 95\% CI, 6.20 to 9.01) (Fig. 3). There was no significant heterogeneity of the increase observed in the average blood flow velocity of the left vertebral artery amongst the studies $\left(\chi^{2}=0.32 ; \mathrm{P}=0.57 ; \mathrm{I}^{2}=0 \%\right)$, leading to application of the fixed-effect model. Statistical results revealed a statistically significant increase in the average blood flow velocity of the left vertebral artery (OR, 6.17; 95\% CI, 5.22 to 7.13) (Fig. 4). However, significant heterogeneity was reported in the change to blood flow velocity of the right vertebral artery amongst the studies $\left(\chi^{2}=12.59 ; \mathrm{P}=0.0004\right.$; $\mathrm{I}^{2}=92 \%$ ). In order to identify the cause of the heterogeneity, a sensitivity analysis and an additional subgroup analysis were performed; however, they were unable to indicate the etiology of the heterogeneity, meaning that descriptive analysis was conducted (Fig. 5).
A total of 2 studies $(23,26)$ reported that, following treatment, the average blood flow velocity of the right vertebral artery in the experimental group was $35.62 \pm 3.20$ and $45.18 \pm 2.36 \mathrm{~cm} / \mathrm{s}$; the corresponding control values were $26.10 \pm 3.30$ and $39.49 \pm 2.27 \mathrm{~cm} / \mathrm{s}$, respectively. The difference between the groups in the two trials was statistically significant $(\mathrm{P}<0.05)$.

Adverse effects. Adverse reactions of the experimental and control groups included 396 cases (199 cases reported within the experimental groups and 198 cases in the control groups), reported across 4 trials $(22,24-26)$. Analysis demonstrated that the difference in heterogeneity amongst the trials was not significant $\left(\chi^{2}=4.72 ; \mathrm{P}=0.19 ; \mathrm{I}^{2}=36 \%\right.$ ), and the fixed-effect model was used. There was a statistically significant difference in adverse events between the intervention and control groups 


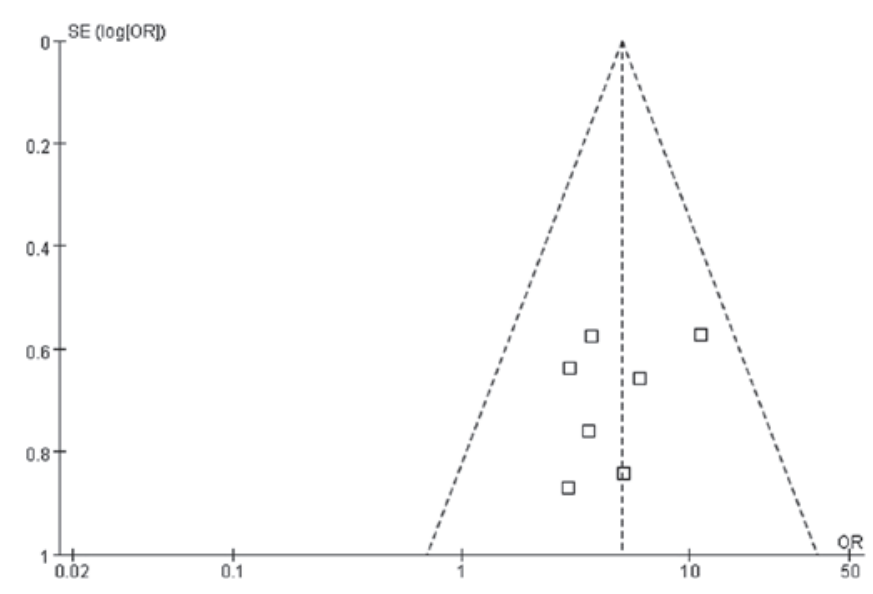

Figure 7. Funnel plot evaluating publication bias.

(OR, $0.75 ; 95 \%$ CI, 0.32 to 1.77 ), suggesting a lower incidence of adverse reactions in the experimental than the control group in the treatment of VBI vertigo (Fig. 6).

Publication bias. A funnel plot was used to detect publication bias (Fig. 7). All 7 studies included in the present meta-analysis were within $95 \% \mathrm{CI}$, and the plot is an inverted funnel shape, with the results predominantly within the middle-lower region of the funnel plot. However, the distribution was slightly asymmetric, which suggests a possible publication bias; however, the publication bias was lower.

\section{Discussion}

The present meta-analysis examined 7 RCTs, including a total of 664 participants, and reviewed clinical studies of puerarin combined with betahistine in the treatment of VBI vertigo, evaluating the safety and efficacy of this co-treatment. The majority of the trials reported that this combination treatment was effective in treating VBI vertigo, evaluated based on the total efficacy rate, the improvements of vertigo symptoms, increased average blood flow velocity of the basilar artery or fewer adverse reactions. The overall methodological quality of the 7 trials was 'moderate' or 'low.' However, to the best of our knowledge a number of the trials included in the present study had not previously been included in meta-analyses, which should enhance the validity of the present evaluation of the effects of puerarin co-treatment with betahistine.

VBI vertigo, caused by ischemia within the vestibular system (40), is a common disease in elderly patients, who are likely to be more vulnerable to its associated risks. Furthermore, VBI vertigo markedly affects the quality of life and health of patients (10). Previous clinical studies have reported that puerarin may improve blood circulation in the neck (28), inhibit platelet aggregation (41), improve the deformability of red blood cells (42) and expand the coronary artery and cerebrovascular system, thereby promoting blood microcirculation (29). Puerarin is therefore widely used in clinical prevention and treatment of a variety of ischemic cardiovascular and cerebrovascular diseases (43).

The results of the present meta-analysis suggested that puerarin and betahistine co-treatment produced positive results in patients with VBI vertigo compared with the control groups, providing support for its clinical application. The present results demonstrated that the total efficacy rate was higher in the experimental groups compared with the control groups. Improvements of the vertigo symptoms and an increased average blood flow velocity of the basilar artery were reported in 2 trials $(23,26)$. However, the small sample size (seven RCTs) and three relatively low-quality studies ( 2 points by Jadad's validated score) may have limited the accuracy of the statistical analyses, which in turn may have reduced the validity of the present study. The current study demonstrated that puerarin combinatorial treatment with betahistine increased the average blood flow velocity of the basilar artery of the patients with VBI. However, due to the prevalence of limiting factors, such as short treatment duration, lack of detail regarding follow-up investigation and small sample sizes, the results of the present study require additional investigation.

A total of 4 studies $(22,24-26)$ reported adverse reactions in the intervention and control groups, which primarily included nausea and abdominal discomfort; however, these occurred at lower incidence in the intervention group. Due to the small sample size and quality issues with the studies, this analysis should be repeated on a greater number of high-quality RCTs.

The present analysis has a number of potential limitations, warranting conservative interpretation. Firstly, the quality of the RCTs included was generally low. The included RCTs claimed randomization, but provided limited description of the methodology underlying this, which may lead to selection bias. Secondly, the majority of the included systematic reviews used the improvement of vertigo and other clinical symptoms as the primary outcomes. However, these studies predominantly lacked long-term follow-up indicators, such as progress and recurrence of vertigo symptoms, which are crucial for determining the overall effect of treatment. Thirdly, all previous RCTs claimed that the puerarin co-treatment with betahistine produced an improvement in vertigo symptoms compared with use of conventional drugs alone. In addition, no negative data was identified, despite extensive searches of unpublished material, which may affect the validity of conclusions in the reviews and lead to publication bias. Furthermore, the safety of integrative medicine using conventional western medicine alongside tradition medicine is unclear (44). References to adverse reactions were limited in numerous RCTs, and no in-depth analysis of their causes was performed, which may affect reliability. Finally, only 7 studies were included in the present meta-analysis, which results in a limitation in the accuracy of statistical analysis. Future analyses should include a larger number of studies.

All RCTs evaluated in the present study were published in mainland China, in Chinese, which are therefore not accessible by the international research community. However, 4 trials $(22,24-26)$ were of high methodological quality, based on high Jadad's validated scores (35), which was determined by trial quality assessment. As the quality of numerous trials included in the present meta-analysis was low, additional high-quality trials are required to thoroughly evaluate the effects of puerarin and betahistine co-treatment of VBI vertigo. Therefore, trials must follow basic guidelines established for reporting clinical trials, such as those in the CONSORT statement, and treatment should be rigorously monitored and reported in future clinical trials (45-49). 
The present meta-analysis provides additional evidence to suggest that puerarin combined with betahistine may reduce vertigo and improve the average blood velocity of the basilar artery, with few adverse side effects, in patients with VBI vertigo. However, the evidence presented in the current study should be interpreted cautiously due to the low quality of a number of the included trials. A greater number of rigorously-designed, large sample, multi-center RCTs are required to fully evaluate the effectiveness of puerarin combined with betahistine in VBI vertigo treatment.

\section{Acknowledgements}

The present study was financially supported by the National Natural Science Foundation of China (grant no. 81473365) and the program of Innovative Research Team of Beijing University of Chinese Medicine (grant no. 2011-CXTD-13).

\section{References}

1. Li JQ, Zhang QJ and Li B: Research progress of diagnosis and treatment of vertebral basilar artery blood supply deficiency. Zhong Wai Yi Xue Yan Jiu 30: 154-156, 2011 (In Chinese).

2. Chang WL: 130 case analysis of diagnosis and treatment of vertebral-basilar artery blood supply insufficiency. Shaanxi Yi Xue Za Zhi 39: 1039-1040, 2010 (In Chinese).

3. Li L, Yu LX and Sui P: Curative effect observation of buflomedil hydrochloride combined with shuxuening in the treatment of posterior circulation ischemic vertigo. Zhongguo Shi Yong Shen Jing Ji Bing Za Zhi 13: 9-11, 2010 (In Chinese).

4. Wang FX, Jiang ZM, Huang ZW, Han F, Jin YL and Qiao XL: EAPA turn neck test and research of prognosis of vertebral-basilar artery blood supply insufficiency. Zhong Feng Yu Shen Jing Ji Bing Za Zhi 1: 376, 1994 (In Chinese).

5. Wang XJ: 50 cases of curative effect observation of puerarin combined with sibelium in the treatment of vertebrobasilar ischemia vertigo. Baiqiuen Jun Yi Xue Yuan Za Zhi 7: 301-302, 2009 (In Chinese).

6. Kang YQ and Li ZY: Clinical analysis of vertebrobasilar ischemia. Di Si Jun Yi Da Xue Jilin Jun Yi Xue Yuan Xue Bao 22: 84-87, 2000 (In Chinese).

7. Wong KH,Li GQ, Li KM, Razmovski-Naumovski V and Chan K: Kudzu root: Traditional uses and potential medicinal benefits in diabetes and cardiovascular diseases. J Ethnopharmacol 134: 584-607, 2011.

8. Zhou YX, Zhang $\mathrm{H}$ and Peng C: Puerarin: A review of pharmacological effects. Phytother Res 28: 961-975, 2014.

9. Shi CY, Zhao LX and Guan HY: 102 cases of puerarin for treatment of vertebral basilar artery insufficiency vertigo. Shaanxi Zhong Yi 12: 1324-1325, 2005 (In Chinese).

10. Yao LY: Curative effect observation of alprostadil combined with shuxuening in the treatment of posterior circulation ischemic vertigo. Zhongguo Shi Yong Shen Jing Ji Bing Za Zhi 15: 56-57, 2012 (In Chinese).

11. Hsu FL, Liu IM, Kuo DH, Chen WC, Su HC and Cheng JT: Antihyperglycemic effect of puerarin in streptozotocin-induced diabetic rats. J Nat Prod 66: 788-792, 2003.

12. Meng QH, Zhang YM and Lou FL: Mechanisms of inflammation in AD rats induced by $\alpha \beta$ and the intervention effect of acetyl puerarin and chitosan phosphatidylcholine. Shandong University, 2013 (In Chinese)

13. Wang XX, Zhang YL and $\mathrm{Wu} \mathrm{J}$ : The effects of puerariae radix on bone mass and bone microarchitecture in ovariectomy mice. Zhongguo Guzhi Shusong Zazhi 14: 349-354, 2008 (In Chinese).

14. Miyazawa M, Sakano K, Nakamura $S$ and Kosaka H: Antimutagenic activity of isoflavone from Pueraria lobata. J Agric Food Chem 49: 336-341, 2001.

15. Sun XH, Ding JP, Li H, Pan N, Gan L, Yang XL and Xu HB: Activation of large-conductance calcium-activated potassium channels by puerarin: The underlying mechanism of puerarin-mediated vasodilation. J Pharmacol Exp Ther 323: 391-397, 2007.
16. Fan LL, Sun LH, Li J, Yue XH, Yu HX and Wang SY: The protective effect of puerarin against myocardial reperfusion injury. Study on cardiac function. Chin Med J (Engl) 105: 11-17, 1992.

17. Xu X,Zhang S,Zhang L, Yan W and Zheng X: The Neuroprotection of puerarin against cerebral ischemia is associated with the prevention of apoptosis in rats. Planta Med 71: 585-591, 2005.

18. Guerra MC, Speroni E, Broccoli M, Cangini M, Pasini P, Minghett A, Crespi-Perellino N, Mirasoli M, Cantelli-Forti G and Paolini M: Comparison between Chinese medical herb Pueraria lobata crude extract and its main isoflavone puerarin antioxidant properties and effects on rat liver CYP-catalysed drug metabolism. Life Sci 67: 2997-3006, 2000.

19. $\mathrm{Yu} \mathrm{Z}$ and $\mathrm{Li} \mathrm{W}$ : Induction of apoptosis by puerarin in colon cancer HT-29 cells. Cancer Lett 238: 53-60, 2006.

20. Zhang Y, Zeng X, Zhang L and Zheng X: Stimulatory effect of puerarin on bone formation through activation of PI3K/Akt pathway in rat calvaria osteoblasts. Planta Med 73: 341-347, 2007.

21. Sun XJ and Dang XR: Curative effect observation of betahistine combined with puerarin in the treatment of vertebrobasilar ischemia vertigo. Di 4 Jun Yi Da Xue Xue Bao 28: 2277, 2007 (In Chinese).

22. Yue YM, Qiao B and Zhang YH: 46 cases analysis of curative effect of puerarin combined with betahistine in the treatment of vertebrobasilar ischemia vertigo. Zhongguo Yi Xue Chuang Xin 10: 53-54, 2013 (In Chinese).

23. Yang MG: Curative effect observation of betahistine combined with puerarin in the treatment of vertebrobasilar ischemia vertigo. Hebei Yi Yao 32: 3170-3171, 2010 (In Chinese).

24. Wang L: Curative effect observation of betahistine combined with puerarin in the treatment of vertebrobasilar ischemia vertigo. Dang Dai Yi Xue 17: 109, 2011 (In Chinese).

25. Xie SB: Curative effect observation of betahistine combined with puerarin in the treatment of vertebrobasilar ischemia vertigo. Shi Yong Yi Xue Za Zhi 24: 2323-2324, 2008 (In Chinese).

26. Guo R: Curative effect of puerarin combined with betahistine in the treatment of 43 cases of vertebrobasilar ischemia vertigo. Zhongguo Shi Yong Shen Jing Ji Bing Za Zhi 15: 61-62, 2012 (In Chinese).

27. Lei LF: Curative effect of puerarin combined with betahistine in the treatment of 43 cases of vertebrobasilar ischemia vertigo. Zhongguo Zhong Yi Ji Zheng 17: 1143-1144, 2008 (In Chinese).

28. Qiao D and Ma YZ: Curative effect observation of puerarin injection in the treatment of vertebrobasilar ischemia vertigo. Shijie Zhong Xi Yi Jie He Za Zhi 3: 163, 2008 (In Chinese).

29. Zhang JR: 60 cases of curative effect observation of puerarin combined with lidocaine in the treatment of vertebrobasilar ischemia vertigo. Xian Dai Zhong Xi Yi Jie He Za Zhi 14: 601, 2005 (In Chinese).

30. Altman DG and Riley RD: Primer: An evidence-based approach to prognostic markers. Nat Clin Pract Oncol 2: 466-472, 2005.

31. Trivella M, Pezzella F, Pastorino U, Harris AL and Altman DG; Prognosis In Lung Cancer (PILC) Collaborative Study Group: Microvessel density as a prognostic factor in non-small-cell lung carcinoma: A meta-analysis of individual patient data. Lancet Oncol 8: 488-499, 2007.

32. Cheung F, Feng Y, Wang N, Yuen MF, Tong Y and Wong VT: Effectiveness of Chinese herbal medicine in treating liver fibrosis: A systematic review and meta-analysis of randomized controlled trials. Chin Med 7: 5, 2012.

33. American Heart Association: Recommendations on stroke prevention, diagnosis, and therapy. Report of the WHO Task Force on Stroke and other Cerebrovascular Disorders. Stroke 20: 1407-1431, 1989.

34. Green S (ed): Cochrane Handbook for Systematic Reviews of Interventions. The Cochrane Collaboration, 2011.

35. Jadad AR, Moore RA, Carroll D, Jenkinson C, Reynolds DJ, Gavaghan DJ and McQuay HJ: Assessing the quality of reports of randomized clinical trials: Is blinding necessary? Control Clin Trials 17: 1-12, 1996.

36. QinF,Liu JY and Yuan JH: Chaihu-Shugan-San, an oriental herbal preparation, for the treatment of chronic gastritis: A meta-analysis of randomized controlled trials. J Ethnopharmacol 146: 433-439, 2013.

37. Petitti DB: Approaches to heterogeneity in meta-analysis. Stat Med 20: 3625-3633, 2001

38. Sutton AJ, Duval SJ, Tweedie RL, Abrams KR and Jones DR: Empirical assessment of effect of publication bias on meta-analyses. BMJ 320: 1574-1577, 2000.

39. Higgins JPT and Green S (eds): Cochrane Handbook for Systematic Reviews of Interventions Version 4.2.6. The Cochrane Collaboration, 2006. 
40. Du YQ and Wang XJ: Ozagrel sodium combined with buflomedil treat vertebrobasilar ischemia vertigo. Dang Dai Yi Xue 15: 133, 2009 (In Chinese).

41. Xiao HX: Pharmacological research status of Pueraria lobata. Shizhen Guo Yi GuoYao 12: 1141, 2000 (In Chinese).

42. Liao ML, Yu J and Zhou M: New progress of Puerarin in clinical application. Xian Dai Zhong Xi Yi Jie He Za Zhi 12: 776, 2003 (In Chinese)

43. Lei BX, Hou XL and Wang JP: Effect of anti-cerebral ischemia and hypoxia of kudzu. Zhongguo Yiyuan Yao Xue Za Zhi 20: 15, 2000 (In Chinese).

44. Yang X, Xiong X, Yang G and Wang J: Chinese patent medicine Xuefu Zhuyu capsule for the treatment of unstable angina pectoris: A systematic review of randomized controlled trials. Complement Ther Med 22: 391-399, 2014.

45. Turner L, Shamseer L, Altman DG, Schulz KF and Moher D: Does use of the CONSORT Statement impact the completeness of reporting of randomised controlled trials published in medical journals? A Cochrane review. Syst Rev 1: 60, 2012.
46. Turner L, Shamseer L, Altman DG, Weeks L, Peters J, Kober T, Dias S, Schulz KF, Plint AC and Moher D: Consolidated standards of reporting trials (CONSORT) and the completeness of reporting of randomised controlled trials (RCTs) published in medical journals. Cochrane Database Syst Rev 11: MR000030, 2012.

47. Xiong X, Yang X, Liu W, Feng B, Ma J, Du X, Wang P, Chu F, Li J and Wang J: Banxia baizhu tianma decoction for essential hypertension: A systematic review of randomized controlled trials. Evid Based Complement Alternat Med 2012: 271462, 2012.

48. Xiong X, Yang X, Feng B, Liu W, Duan L, Gao A, Li H, Ma J, Du X, Li N, et al: Zhen gan xi feng decoction, a traditional Chinese herbal formula, for the treatment of essential hypertension: A systematic review of randomized controlled trials. Evid Based Complement Alternat Med 2013: 982380, 2013.

49. Smith SM, Chang RD, Pereira A, Shah N, Gilron I, Katz NP Lin AH, McDermott MP, Rappaport BA, Rowbotham MC, et al: Adherence to CONSORT harms-reporting recommendations in publications of recent analgesic clinical trials: An ACTTION systematic review. Pain 153: 2415-2421, 2012. 